'Departamento de Especialidades Médicas, Facultad de Medicina, Universidad de La Frontera. Temuco, Chile.

Unidad de Neurología, Hospital Dr. Hernán Henríquez Aravena. Temuco, Chile.

${ }^{3}$ Centro de Investigación en Epidemiología Cardiovascular y Nutricional (EPICYN), Universidad de La Frontera. Temuco, Chile. ${ }^{4}$ Departamento de Salud

Pública, Facultad de Medicina, Universidad de La Frontera. Temuco, Chile.

${ }^{5}$ Hospital Intercultural de Nueva Imperial. Temuco, Chile. ${ }^{\mathrm{a} M S c}$.

${ }^{b}$ Nutricionista.

'PhD en Salud Pública.

${ }^{d}$ Interno de Medicina,

Universidad de La Frontera. Temuco, Chile.

Trabajo no recibió financiamiento. Los autores declaran no tener conflictos de interés.

Recibido el 9 de octubre de 2018, aceptado el 20 de junio de 2019.

Correspondencia a: Álvaro Soto Venegas Departamento de Especialidades Médicas, Facultad de Medicina, Universidad de La Frontera. Francisco Salazar \# 01145 , Temuco, Chile. alvaro.soto@ufrontera.cl

\section{Tiempos de llegada de pacientes con ataque cerebrovascular en un hospital regional de alta complejidad}

\author{
ÁLVARO SOTO V. ${ }^{1,2,3, a}$, GLADYS MORALES I..$^{3,4, a, b, c}$, \\ GONZALO ECHEVERRÍA V. ${ }^{5}$, MARÍA BELÉN COLINAS G. ${ }^{\mathrm{d}}$, \\ PEDRO CANALES O. ${ }^{d}$, DANIELA CONTRERAS B. ${ }^{d}$
}

\section{Arrival times of patients with stroke to a public hospital}

Background: Stroke is a time-dependent emergency. Most patients with acute ischemic stroke are excluded from reperfusion therapies due to late consultation. Aims: To estimate the arrival times of patients with stroke to the Emergency Room (ER) of a public hospital. To identify factors associated with early consultation. Material and Methods: A convenience sample, 583 patients aged $71 \pm 13$ years (55\% males) consulting for stroke at an emergency room was analyzed in terms of delay between onset of symptoms and arrival to the ER, demographics and etiology of stroke. Results: The admission diagnoses were ischemic stroke in 76\%, intracerebral hemorrhage in 12\%, transient ischemic attack in $9 \%$ and subarachnoid hemorrhage in 3\%. The median time of arrival was 8 hours and 11 minutes after the onset of symptoms. Nineteen percent of consultations for ischemic stroke occurred within 3 hours of symptom onset, and 38\% within 6 hours. In the logistic regression analysis, having an address near the hospital and the severity of stroke were associated with early consultation with a combined odds ratio of 5.97 (95\% confidence intervals 3.23-11.04). Conclusions: There were significant differences in the arrival times of patients with stroke. Only a low proportion of patients with ischemic stroke consulted within the window for reperfusion therapies. Severe strokes and living near the hospital were associated with early consultation.

(Rev Med Chile 2019; 147: 1154-1158)

Key words: Emergency Services, Medical; Hospitals; Neurologists; Stroke; Thrombectomy; Trombolytic Therapy.
E $l$ ataque cerebrovascular (ACV) constituye el principal motivo de evaluación por neurólogo en el Servicio de Urgencia $(\mathrm{SU})^{1}$. El ACV es tiempo-dependiente. El manejo adecuado debe instaurarse lo antes posible para minimizar secuelas funcionales significativas y permanentes ${ }^{2}$. En el infarto cerebral, por cada 15 min de disminución del retraso para la trombolisis se obtiene $4 \%$ de aumento en la probabilidad de un buen desenlace funcional ${ }^{3}$.

En una revisión sistemática de Evenson y cols. de 2009 se estimó una mediana de 3-4 h entre el inicio de los síntomas y la llegada al SU, con 6-92\% de los sujetos consultando dentro de $3 \mathrm{~h}^{4}$. Por otra parte, una revisión reciente encontró que la mediana del tiempo de inicio de los síntomas y la llegada a urgencia, y el porcentaje de pacientes con $\mathrm{ACV}$ que consultan antes de $3 \mathrm{~h}$ se han mantenido estables en las últimas dos décadas ${ }^{5}$.

Dentro de los factores más frecuentemente asociados con una llegada precoz al SU de pacientes con ACV agudo destacan: transporte en 
ambulancia, gravedad del ACV, sintomatología, subtipo de ACV, comorbilidades, comportamiento o percepción del paciente o familiar al inicio del cuadro y horario de inicio los síntomas 5 . Dentro de los factores asociados a retraso para la consulta se incluyen: sexo, raza, medio de llegada e historia de enfermedad cardiovascular ${ }^{6}$.

El Hospital Dr. Hernán Henríquez Aravena (HHHA) de Temuco cuenta con neurólogos presenciales $24 / 7$ en el SU desde julio de $2013^{1,7}$. Nuestro establecimiento tiene 730 camas, es el único hospital de alta complejidad de la Región de La Araucanía, y atiende a una población beneficiaria de aproximadamente 800 mil habitantes ${ }^{8}$. El Servicio de Salud Araucanía Sur cuenta, además, con cuatro hospitales de mediana complejidad (nodos) y ocho hospitales de baja complejidad. El HHHA también es centro de derivación de urgencias neurológicas provenientes del Servicio de Salud Araucanía Norte. La Región de La Araucanía tiene una superficie de $31.842,3 \mathrm{~km}^{2}$, lo que representa $4,2 \%$ del territorio continental e insular del país 9 La ruralidad de la región alcanza a 29,1\% y es la segunda a nivel nacional ${ }^{10}$. La conurbación Temuco-Padre Las Casas (PLC) tiene aproximadamente 360 mil habitantes ${ }^{10}$. Los puntos de la región más alejados de Temuco son Lonquimay y Curarrehue a 166,7 y $141,3 \mathrm{~km}$, respectivamente.

El presente trabajo se realizó con los siguientes objetivos: estimar los tiempos de llegada de los pacientes con ACV e identificar los factores asociados a una consulta precoz.

\section{Material y Método}

El estudio fue prospectivo y observacional. Se seleccionó una muestra no probabilística, por conveniencia, de las consultas por ACV realizadas en el Turno $\mathrm{N}^{\circ} 1$ del SU-HHHA, entre enero de 2016 y diciembre de 2017.

Se consideraron los antecedentes sociodemográficos, hora de inicio de los síntomas y hora de ingreso al SU. En el caso que el paciente despertara con el déficit se consideró como inicio la última hora en que el paciente fue visto en su condición basal (last known well). Se consideró como mapuche a los sujetos con dos apellidos en esa lengua. Se registró comuna de residencia, procedencia (urbano/rural) y medio de llegada (ambulancia, propios medios).
Los diagnósticos se agruparon en: ataque isquémico transitorio (AIT), infarto cerebral (IC), hemorragia intracerebral (HIC) y hemorragia subaracnoídea (HSA). La gravedad del ACV fue estimada con la escala del National Institutes of Health Stroke Scale (NIHSS) ${ }^{11}$. Se utilizó 7 puntos en la escala de NIHSS como punto de corte por su capacidad para predecir una oclusión arterial ${ }^{12}$.

Las variables continuas se describieron con medidas de tendencia central y dispersión, promedio \pm desviación estándar (DE) o medianas con percentiles 25-75 (p25-p75). Se utilizó test $\chi^{2}$ para evaluar la asociación entre la variable de resultado (llegada $<3 \mathrm{~h}$ ) y las variables de control: sexo, edad $\geq 65$ años, residencia en Temuco-PLC, procedencia, etnia, gravedad del ACV y medio de llegada. Finalmente, se creó un modelo de regresión logística para estimar la fuerza de la asociación entre las variables de control y la variable de resultado. El análisis estadístico se realizó con el software STATA 14.0.

\section{Aspectos éticos}

La realización de este estudio fue aprobada por el Comité Ético Científico del Servicio de Salud Araucanía Sur.

\section{Resultados}

Se registraron 584 consultas por ACV agudo. Los diagnósticos fueron: IC $=75,9 \%$; HIC $=$ $11,8 \%$; AIT $=9,1 \%$ y HSA $=3,3 \%$. Las características clínicas y sociodemográficas de los pacientes se describen en la Tabla 1.

La mediana del tiempo de llegada fue de $8 \mathrm{~h} \mathrm{y}$ $11 \mathrm{~min}$ (491 min). El 21,8\% de los pacientes consultó dentro de $3 \mathrm{~h}$. Solo $19,4 \%$ de las consultas por infarto cerebral se realizaron dentro de $3 \mathrm{~h}, \mathrm{y}$ $38,2 \%$ dentro de $6 \mathrm{~h}$. Los tiempos de llegada según el tipo de ACV se describen en la Tabla 2.

Dentro de las variables asociadas a una llegada rápida al SU $(<3 \mathrm{~h})$ destacaron: residencia en Temuco-PLC, origen urbano, llegada en ambulancia y gravedad del ACV definido por un puntaje $\geq 7$ en la escala de NIHSS (Tabla 3 ).

En el modelo de regresión logística para llegada menor a $3 \mathrm{~h}$, las variables domicilio en Temuco-PLC y la gravedad del ACV alcanzaron significación estadística con un OR combinado de 5,97 (IC 95\%: 3,23-11,04) (Tabla 4). 
Tabla 1. Características clínicas y biodemográficas de los pacientes con ataque cerebrovascular

\begin{tabular}{|lccccc|}
\hline Parámetros & IC & HIC & AIT & HSA & Total \\
$\mathrm{n}(\%)$ & $443(75,9)$ & $69(11,8)$ & $53(9,1)$ & $19(3,3)$ & $584(100)$ \\
\hline Hombres (\%) & 56,2 & 49,3 & 56,6 & 47,4 & 55,1 \\
\hline Edad (promedio, DE) & $71,1 \pm 13,1$ & $67,1 \pm 13,8$ & $73,2 \pm 11,7$ & $65,2 \pm 16,7$ & $70,7 \pm 13,3$ \\
$\geq 65$ años (\%) & 70,2 & 56,5 & 79,3 & 47,4 & 68,7 \\
Temuco-PLC (\%) & 41,8 & 33,3 & 45,3 & 57,9 & 41,6 \\
Ruralidad (\%) & 30 & 39,1 & 15,1 & 15,8 & 29,3 \\
Etnia mapuche (\%) & 15,8 & 30,4 & 3,8 & 21,1 & 16,6 \\
NIHSS (mediana, $\mathrm{P}_{25}-\mathrm{P}_{75}$ ) & $4(2-9)$ & $9(3-19)$ & 0 & $3(0-42)$ & $3(1-9)$ \\
\hline
\end{tabular}

IC: infarto cerebral; HIC: hemorragia intracerebral; AIT: ataque isquémico transitorio; HSA: hemorragia subaracnoidea; PLC: Padre Las Casas; NIHSS = National Institutes of Health Stroke Scale.

Tabla 2. Tiempos de inicio-llegada según tipo de ataque cerebrovascular

\begin{tabular}{|c|c|c|c|c|c|c|}
\hline Tipo de ACV & $\%$ & Tiempo de llegada (mediana, $\mathbf{P}_{25}-\mathbf{P}_{75}$ ) & $\begin{array}{c}<1 \mathrm{~h} \\
(\%)\end{array}$ & $\begin{array}{c}<3 \text { h } \\
(\%)\end{array}$ & $\begin{array}{c}<6 \mathrm{~h} \\
(\%)\end{array}$ & $\begin{array}{c}<24 \mathrm{~h} \\
(\%)\end{array}$ \\
\hline Infarto cerebral & 75,9 & 9 h 53' $^{\prime} \quad(3$ h 39' -28 h 38') & 2,7 & 19,4 & 38,2 & 69,8 \\
\hline Hemorragia intracerebral & 11,8 & 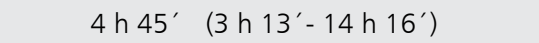 & 1,5 & 17,4 & 58 & 82,6 \\
\hline Ataque isquémico transitorio & 9,1 & 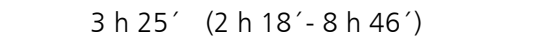 & 7,6 & 37,7 & 67,9 & 88,7 \\
\hline Hemorragia subaracnoídea & 3,3 & 3 h $48^{\prime} \quad\left(1\right.$ h $53^{\prime}-13$ h $\left.41^{\prime}\right)$ & 15,8 & 47,4 & 63,2 & 84,2 \\
\hline Total & 100 & 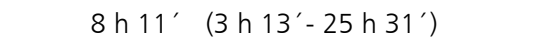 & 3,4 & 21,8 & 44 & 73,5 \\
\hline
\end{tabular}

Tabla 3. Porcentaje de inicio-llegada menor a $3 \mathbf{~ h}$, según características biodemográficas, medio de llegada y severidad del ataque cerebrovascular

\begin{tabular}{|c|c|c|}
\hline Variables & Llegada < 3 h (\%) & Valor $\mathbf{p}$ \\
\hline $\begin{array}{l}\text { Sexo } \\
\text { Masculino } \\
\text { Femenino }\end{array}$ & $\begin{array}{l}20,8 \\
22,9\end{array}$ & 0,542 \\
\hline $\begin{array}{l}\text { Edad } \\
\qquad \begin{array}{l}\quad 65 \text { años } \\
<65 \text { años }\end{array}\end{array}$ & $\begin{array}{l}21,5 \\
22,4\end{array}$ & 0,795 \\
\hline $\begin{array}{l}\text { Residencia } \\
\text { Temuco-PLC } \\
\text { Otra }\end{array}$ & $\begin{array}{l}32,9 \\
13,8\end{array}$ & 0,000 \\
\hline $\begin{array}{l}\text { Origen } \\
\text { Urbano } \\
\text { Rural }\end{array}$ & $\begin{array}{l}25,2 \\
13,5\end{array}$ & 0,002 \\
\hline $\begin{array}{l}\text { Etnia } \\
\text { Mapuche } \\
\text { No mapuche }\end{array}$ & $\begin{array}{l}14,4 \\
23,2\end{array}$ & 0,056 \\
\hline $\begin{array}{l}\text { Medio de llegada } \\
\text { Ambulancia } \\
\text { Propios medios }\end{array}$ & $\begin{array}{c}20 \\
29,2\end{array}$ & 0,032 \\
\hline $\begin{array}{l}\text { Severidad } \\
\text { NIHSS } \geq 7 \\
\text { NIHSS }<7\end{array}$ & $\begin{array}{l}30,4 \\
16,6\end{array}$ & 0,000 \\
\hline
\end{tabular}

PLC: Padre Las Casas; NIHSS = National Institutes of Health Stroke Scale.
Tabla 4. Modelo de regresión logística: Efecto de las variables de control sobre la variable de respuesta llegada en menos de $3 \mathbf{h}$

\begin{tabular}{|lccc|}
\hline Variables & Odds ratio & Valor p & IC 95\% \\
\hline Severidad & 2,3 & 0,000 & $1,51-3,51$ \\
\hline Medio de llegada & 0,9 & 0,696 & $0,52-1,54$ \\
\hline Sexo & 0,89 & 0,592 & $0,59-1,35$ \\
Edad & 0,9 & 0,648 & $0,58-1,41$ \\
Residencia & 2,6 & 0,000 & $1,61-4,18$ \\
Ruralidad & 0,63 & 0,089 & $0,37-1,07$ \\
\hline
\end{tabular}

\section{Discusión}

En nuestro estudio encontramos que la mediana del tiempo de llegada de los pacientes con ACV agudo fue de $8 \mathrm{~h}$ y $11 \mathrm{~min}$. En el subgrupo de IC, 19,4\% de los sujetos consultó dentro de 3 h (ventana terapéutica para trombolisis endovenosa) y $38,2 \%$ dentro de $6 \mathrm{~h}$ (ventana para trombectomía mecánica $)^{13-15}$.

El tiempo inicio-llegada por ACV agudo en 
nuestro estudio fue significativamente mayor que el comunicado por Evenson y cols (mediana de 3-4 h) ${ }^{4}$. La Región de La Araucanía tiene 17,2\% de pobreza por ingresos, el doble de la tasa nacional $(8,6 \%)^{16}$. También posee $34,3 \%$ de pueblos originarios ( $12,8 \%$ nacional); 9,9 años promedio de escolaridad ( $11,1 \%$ nacional) y $29,1 \%$ de rura$\operatorname{lidad}^{10}$. Estos indicadores pueden estar asociados a un mayor desconocimiento de los síntomas de ACV y a mayores barreras de acceso al SU. Por otra parte, encontramos que tener domicilio en la conurbación Temuco-PLC, el origen urbano, la llegada por propios medios y la gravedad del ACV (NIHSS $\geq 7$ ) se asociaron a un tiempo de llegada menor a $3 \mathrm{~h}$.

El efecto de la edad sobre el tiempo de llegada es contradictorio. Por ejemplo, Lacy comunicó que los pacientes de 65 a 74 años consultaban antes que los menores de 55 años $^{6}$, mientras que Fogelholm concluyó que tener más de 70 años se asociaba a un retraso en la consulta ${ }^{17}$. En nuestro estudio no encontramos asociación entre edad $\geq 65$ años y tiempo para la consulta.

En nuestro trabajo concluimos que llegar por propios medios se asoció a un menor tiempo de llegada, comparado con acceder al hospital en ambulancia, lo que es discordante con varias publicaciones ${ }^{5,6,18}$. Esto pudiera explicarse por el déficit de ambulancias en el sistema público chileno, comparado con países desarrollados. Otro factor a destacar es la gravedad del ACV. Los sujetos con ACV grave tienden a consultar antes ${ }^{6,19,20}$.

En nuestra serie, solo 19,4\% de las consultas por infarto cerebral se realizaron dentro de $3 \mathrm{~h}$, lo que constituye la principal causa de la baja tasa de trombolisis en nuestro hospital, que alcanza a $6 \%{ }^{8}$.

Como fortalezas de nuestro estudio podemos mencionar que, según nuestro conocimiento, este es el primer trabajo realizado en Chile que aporta información sobre los tiempos de llegada de pacientes con ACV agudo. También destacamos el elevado número de pacientes incluidos (584 consultas) y el carácter prospectivo del estudio. Dentro de las debilidades podemos incluir que se trata de un registro unicéntrico, con atenciones realizadas por un solo neurólogo y que no se informa acerca del grado de conocimiento de los sujetos respecto a los síntomas de ACV agudo ni del itinerario seguido por los pacientes antes de llegar al SU-HHHA.
Podemos concluir que existen diferencias significativas en los tiempos de llegada de los pacientes con ACV. Solo una baja proporción de los pacientes con infarto cerebral consultan dentro de la ventana terapéutica para terapias de reperfusión. Sugerimos que los esfuerzos para disminuir los tiempos de llegada de los pacientes con ACV debieran enfocarse en aumentar el reconocimiento de síntomas de ACV en la población y en disminuir las barreras de acceso al servicio de urgencia para una pronta evaluación por neurólogo(a).

Agradecimientos: Nuestros agradecimientos al Dr. Sergio Muñoz Navarro por su asesoría para el análisis estadístico.

\section{Referencias}

1. Soto A, Morales G, Pollak D, Jara V. Análisis de las consultas neurológicas en el Servicio de Urgencia de un hospital terciario. Rev Chil Neuro-Psiquiat 2016; 54 (2): 93-101.

2. Lees KR, Bluhmki E, von Kummer R, Brott TG, Toni $\mathrm{D}$, Grotta JC, et al. Time to treatment with intravenous alteplase and outcome in stroke: an updated pooled analysis of ECASS, ATLANTIS, NINDS, and EPITHET trials. Lancet 2010; 375: 1695-703.

3. Saver JL, Fonarow GC, Smith EE, Reeves MJ, Grau-Sepulveda MV, Pan W, et al. Time to Treatment With Intravenous Tissue Plasminogen Activator and Outcome From Acute Ischemic Stroke. JAMA 2013; 309 (23): 2480-8.

4. Evenson KR, Foraker RE, Morris DL, Rosamond WD. A comprehensive review of prehospital and in-hospital delay times in acute stroke care. Int J Stroke 2009; 4: 187-99.

5. Pulvers JN, Watson JDG. If time is brain where is the improvement in prehospital time after stroke? Front Neurol 2017; 8: 617.

6. Lacy CR, Suh DC, Bueno M, Kostis JB. Delay in Presentation and Evaluation for Acute Stroke: Stroke Time Registry for Outcomes Knowledge and Epidemiology (S.T.R.O.K.E.). Stroke 2001; 32: 63-9.

7. Soto A, Morales G, Vega C, Echeverría G, Colinas MB, Canales $\mathrm{P}$, et al. Tiempos de atención de urgencias neurológicas en un hospital regional de alta complejidad. Rev Med Chile 2018; 146: 885-9.

8. Soto A, Morales G, Grandjean M, Pollak D, Del Castillo C, García P, et al. Evolución del protocolo de trombolisis endovenosa en ataque cerebrovascular isquémico 
agudo: 4 años de experiencia en el Hospital Doctor Hernán Henríquez Aravena de Temuco- Chile. Rev Med Chile 2017; 145: 468-75.

9. Instituto Nacional de Estadísticas-Chile. Compendio Estadístico Región de La Araucanía 2017. Disponible en: http://www.inearaucania.cl/archivos/files/pdf/Ediciones\%20Especiales/Compendio\%202017.pdf [Consultado el 5 de marzo de 2019].

10. Instituto Nacional de Estadísticas-Chile. Resultados Censo 2017. Disponible en: http://www.censo2017.cl/ wp-content/uploads/2018/05/presentacion_de_la_segunda_entrega_de_resultados_censo2017.pdf [Consultado el 15 de mayo de 2018].

11. Brott T, Adams HP Jr, Olinger CP, Marler JR, Barsan WG, Biller J, et al. Measurements of acute cerebral infarction: a clinical examination scale. Stroke 1989; 20: 864-70.

12. Olavarría VV, Delgado I, Hoppe A, Brunser A, Cárcamo D, Díaz-Tapia V, et al. Validity of the NIHSS in predicting arterial occlusion in cerebral infarction is time-dependent. Neurology 2011; 76: 62-8.

13. Wardlaw JM, Murray V, Berge E, del Zoppo G, Sandercock P, Lindley RL, et al. Recombinant tissue plasminogen activator for acute ischaemic stroke: an updated systematic review and meta-analysis. The Lancet 2012; 379 (9834): 2364-72.

14. Ministerio de Salud de Chile. Guía clínica AUGE. Accidente Cerebrovascular Isquémico en personas de
15 años y más. Serie de las guías clínicas de MINSAL. 2013. Disponible en: http://www.minsal.cl/portal/url/ item/7222754637e58646e04001011f014e64.pdf [Consultado el 23 de junio de 2018].

15. Badhiwala JH, Nassiri F, Alhazzani W, Selim MH, Farrokhyar F, et al. Endovascular Thrombectomy for Acute Ischemic Stroke: A Meta-analysis. JAMA 2015; 314 (17): 1832-43.

16. Gobierno de Chile. Ministerio de Desarrollo Social, Encuesta de Caracterización Socioeconómica Nacional (Casen) 2017. Situación de pobreza. Disponible en: http://observatorio.ministeriodesarrollosocial.gob.cl/ casen-multidimensional/casen/docs/Resultados_pobreza_Casen_2017.pdf [Consultado el 5 de marzo de 2019].

17. Fogelholm R, Murros K, Rissanen A, Ilmavirta M. Factors delaying hospital admission after acute stroke. Stroke 1995; 27: 398-400.

18. Faiz KW, Sundseth A, Thommessen B, Rønning OM. Prehospital delay in acute stroke and TIA. Emerg Med J 2013; 30: 669-74.

19. Jorgensen HS, Nakayama H, Reith J, Raaschou HO, Olsen TS. Factors delaying hospital admission in acute stroke: the Copenhagen stroke study. Neurology 1996; 47: 383-7.

20. Bouckaert M, Lemmens R, Thijs V. Reducing prehospital delay in acute stroke. Nat Rev Neurol 2009; 5: 47783. 